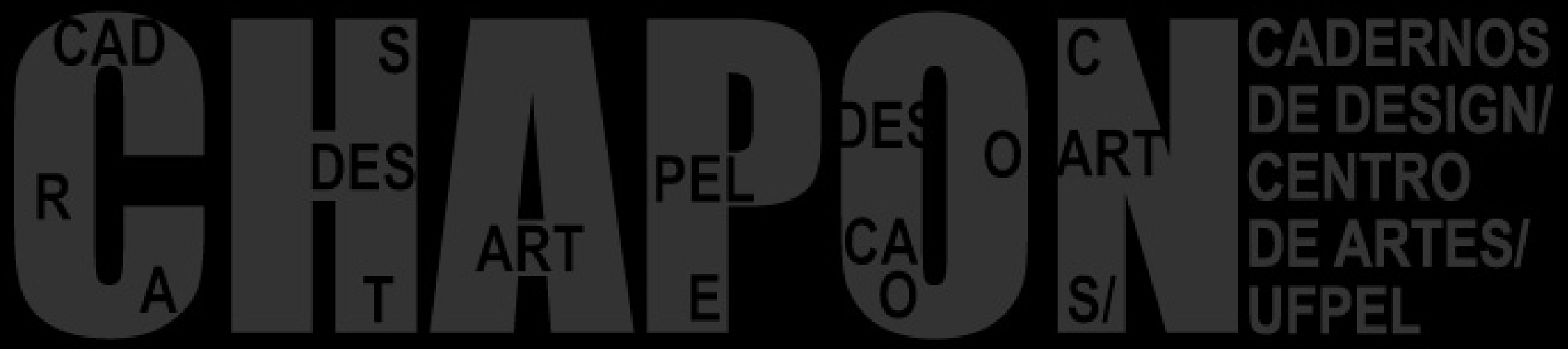

LINGUAGEN METODOLOGIAS TEORIAS VISUALIDADES 


\section{O GUIA GLOBAL DA CIDADE AMIGA DO IDOSO: SUSTENTABILIDADE E CONTRIBUIÇÕESDODESIGN}

Camila Feldberg Porto - UEMG | feldbergc@gmail.com Edson José Carpintero Rezende - UEMG | edson.carpintero@gmail.com 
O aumento da população idosa em todo o mundo e o processo de urbanização pelo qual as cidades passam levaram a Organização Mundial da Saúde (OMS) a criar o Guia Global da Cidade Amiga do Idoso. No guia constam questões trazidas pelos idosos das primeiras 33 cidades onde ele foi aplicado. Neste estudo, foram considerados os aspectos da sustentabilidade social e as possíveis contribuições do design. Por meio de uma revisão da literatura sobre sustentabilidade e design, realizou-se uma análise dos tópicos "espaços abertos e prédios", "transporte" e "moradia" dentre os oito preconizados pelo guia e foi possível identificar que as necessidades dos idosos refletem um desejo por sustentabilidade que pode ser amparado pelo design universal.

Palavras-chave: cidade amiga do idoso; sustentabilidade social; design universal.
The increase of elderly population in the whole world and the urbanization process through which the cities go have taken the World Health Organization (WHO) to create the Age-Friendly Cities Guide. In this guide there are issues brought up by elders from the first 33 cities where the guide was used. In this study, we considered social sustainability aspects and design contributions. By means of a literature review on sustainability and design, we analyzed three topics out of the eight topics presented by the guide: "outdoor spaces and buildings", "transportation" and "housing". We identified that the elderly's necessities reflect a desire for sustainability that can be supported by universal design.

Keywords: age-friendly cities; social sustainability; universal design. 
O Brasil vivencia um processo de envelhecimento da sua população e se acredita que, até 2025 , o país apresente a sexta população mais idosa (com mais de sessenta anos), com uma representação de $15 \%$ da população total. Esta realidade vem sendo possível devido a melhorias e avanços científicos na área da saúde, bem como na redução das taxas de mortalidade e fecundidade (OLIVEIRA et al., 2016). Outra previsão é de que os idosos representarão $19 \%$ dos brasileiros em 2050 (NASRI, 2008). Some-se a isto o fato de os cidadãos estarem mais urbanizados, ou seja, morarem em meios urbanos (OMS, 2008). No Brasil, 84,35\% da população reside nestas áreas (JORDÃO; SILVA, 2014).

De um modo geral, o ambiente tem forte influência sobre o bem-estar das pessoas, suas emoções e capacidades. Isto é ainda mais relevante para o idoso, pois com o passar dos anos ele cria elos mais fortes com o espaço onde vive, ao passo que suas formas de interação com o local vão perdendo a intensidade, dados os declínios decorrentes do processo de envelhecimento. Os ambientes onde os idosos moram e convivem, portanto, devem ser adaptados às suas necessidades, no intuito de garantir que eles tenham uma vida saudável e independente (PORTO; REZENDE, 2016). Com a maior parte da população residindo em meios urbanos, surge a necessidade de repensar as cidades para melhor atender à sua população. Assim, os idosos precisam de ambientes que lhes proporcionem bem-estar, segurança e envolvimento social. Por esta razão a Organização Mundial de Saúde (OMS) e a Organização das Nações Unidas (ONU) defendem a importância de a cidade oferecer estrutura e oportunidades para que seus residentes se mantenham bem e produtivos (OMS, 2008; PEDRO et al., 2013). Também é defendido pela OMS o conceito de envelhecimento ativo, que se refere a um processo que: ressalta a independência, visando a valorização do idoso por seu próprio potencial físico, social e mental; garante que ele prolongue a sua participação na sociedade; prevê que a sociedade respeite as suas necessidades, capacidades e desejos, enquanto lhes garante segurança, proteção e cuidados adequados (OMS, 2005).

Tendo em vista o envelhecimento ativo e a crescente urbanização, a OMS desenvolveu o Guia Global da Cidade Amiga do Idoso. Ele fornece diretrizes para classificar uma cidade ou região como amiga do idoso ou não e o que pode ser melhorado dentro de oito tópicos principais: espaços abertos e prédios; transporte; moradia; participação social; respeito e inclusão social; participação cívica e emprego; comunicação e informação e apoio comunitário e serviços de saúde (OMS, 2008).

Na busca por maior qualidade de vida, bem-estar e 
ambientes mais amigáveis aos idosos, é necessário que se alcance um desenvolvimento sustentável. Os três primeiros tópicos do guia (espaços abertos e prédios, transporte e moradia) são, de todos, os que apresentam maior quantidade de interfaces concretas entre os idosos e o que a cidade tem a ofertar. Os demais tópicos (participação social; respeito e inclusão social; participação cívica e emprego; comunicação e informação; e apoio comunitário e serviços de saúde) são muito, mas não exclusivamente, relacionados a serviços e, por isso, seu contato com a população idosa é mais subjetivo. Por esta razão, esta pesquisa buscou estudar os três primeiros tópicos abordados pelo Guia Global da Cidade Amiga do Idoso e fazer uma relação com a sustentabilidade e o design com foco em soluções mais concretas. Objetivou-se identificar vertentes do design que possam fornecer contribuições para as questões mencionadas no guia.
2.

GUIA GLOBAL DA CIDADE AMIGA DO IDOSO
Partindo do cenário de crescimento da população idosa, principalmente nas regiões urbanas, e da proposta de envelhecimento ativo, a OMS desenvolveu, em 2006, o Protocolo de Vancouver. Ele foi aplicado em 33 cidades ao redor do mundo e teve a finalidade de guiar a pesquisa que levou à criação do Guia Global da Cidade Amiga do Idoso, em 2007. As cidades participantes abrangeram tanto países desenvolvidos quanto em desenvolvimento, incluindo cidades grandes, como Tóquio (Japão) e Londres (Inglaterra), e cidades pequenas, como Mayagüez (Porto Rico) e Montego Bay (Jamaica). Dentre elas também participaram Rio de Janeiro (Brasil) e La Plata (Argentina), que foram as únicas da América do Sul (OMS, 2008). Vale ressaltar que, desde que o guia foi desenvolvido, pesquisadores vêm estudando e aplicando as diretrizes em outras localidades. No Brasil, foi possível identificar exemplos de pesquisas realizadas no Rio Grande do Sul, com o estudo de Rauth, Santos e Pedde (2012) nas cidades de Novo Hamburgo, Veranópolis e Santa Rosa, e em São Paulo, com os estudos de Graeff, Domingues e Bestetti (2012) no bairro do Brás na capital paulista e de Pedro et al. (2013) na cidade de Franca.

O protocolo de Vancouver disponibiliza algumas diretrizes para avaliar cidades ou regiões como amigas do idoso ou não, além de detectar questões a serem 
trabalhadas. Tendo o bem-estar do idoso como principal norte, o protocolo permite a compreensão dos pontos fortes e fracos da cidade e dá voz à população idosa e aos profissionais que se relacionam com ela, no intuito de gerar melhorias. Não existe um local ótimo a ser tomado como exemplo, pois cada cidade tem as suas particularidades. Por isso, ressalta-se que o guia propõe um instrumento de auto-avaliação e de mapeamento para a evolução dos melhoramentos (OMS, 2008).

Os temas abordados seguem um checklist dividido em oito tópicos principais: (1) espaços abertos e prédios; (2) transporte; (3) moradia; (4) participação social; (5) respeito e inclusão social; (6) participação cívica e emprego; (7) comunicação e informação; e (8) apoio comunitário e serviços de saúde. A coleta de dados, que se dá por meio da técnica de grupos focais, ocorre da base em direção ao topo (bottom-up), ou seja, parte diretamente das experiências dos idosos e dos cuidadores, que são os principais beneficiários do guia. Busca-se, também, as opiniões dos prestadores de outros serviços, públicos e privados, que são utilizadas para complementar as informações iniciais. Os resultados são passados dos coordenadores do projeto para o público, a fim de discutir a sua relevância e buscar soluções. Em seguida, eles são passados para os tomadores de decisão e, assim, as ações poderão começar a ser realizadas por meio de políticas públicas. O guia sugere a formulação de uma agenda de ações, tornando possível acompanhar os resultados e avaliar a qualidade que a cidade passa a ter como amiga do idoso (OMS, 2008).
Assim, os resultados representam as opiniões das principais partes interessadas, considerando-se as limitações físicas e emocionais dos idosos. Isto contribui para uma nova percepção de como o ambiente interfere no cotidiano destas pessoas e contribui para a identificação de oportunidades de melhoria (BESTETTI; GRAEFF; DOMINGUES, 2012). Além disso, é interessante observar que a metodologia proposta é adaptável conforme o contexto local e embasamento teórico utilizado (GRAEFF; DOMINGUES; BESTETTI, 2012).

Monteiro, Zazzetta e Araújo Júnior (2015) acreditam que os projetos de cidade amiga do idoso levam a cidades mais sustentáveis, porque contribui para o envelhecimento ativo da população, melhora a qualidade de vida e estimula a elaboração de políticas públicas, enquanto favorece a criação de ambientes e serviços voltados para os idosos e seu bem estar. 
3. SUSTENTABILIDADE
Sustentabilidade é um assunto contemporâneo que se relaciona com as perspectivas presentes e tem a responsabilidade de pensar nas necessidades e objetivos das gerações futuras (BARBOSA, 2008). Estabelecer um único conceito para o tema é uma tarefa complexa. Os termos sustentabilidade e desenvolvimento sustentável aparecem com frequência na literatura, mas podem sofrer distinções e serem analisados sob a ótica de diferentes áreas do conhecimento. Considera-se que a sustentabilidade signifique, etimologicamente, a qualidade do que é "passível de sustentação" (MONTEIRO; ZAZZETTA; ARAÚJO JÚNIOR, 2015). A sua presença na sociedade atual é cada vez mais reconhecida e a complexidade dos seus conceitos requer uma visão holística do mundo e do lugar do ser humano nele (CHICK, 2012).

Uma das práticas de sustentabilidade é ter como foco o bem estar, a acessibilidade e indicadores de sustentabilidade econômica e ambiental. Isto seria responsável por favorecer um consumo consciente, no qual a qualidade teria mais valor que a quantidade (ALVES, 2006).

Apesar das suas diversas definições, as principais estão relacionadas à satisfação ambiental, econômica e social. Estas três são entendidas como dimensões da sustentabilidade no modelo Triple Bottom Line, de John Elkington, cunhado em 1994, com a intenção de expressar a expansão que se esperava que a agenda de sustentabilidade tivesse na época. Este modelo é largamente aceito em meios acadêmicos e empresariais. Entretanto, vale ressaltar que a dimensão social é, ainda, a menos aprofundada das três (ELKINGTON, 2004; LOURENÇO; CARVALHO, 2013).

Para Faria (2014), a sustentabilidade social é representada pela melhora na qualidade de vida das pessoas, pela distribuição de renda igualitária, redução de diferenças sociais e maior participação e organização da população. Similarmente, Barbosa (2008) explica que ela está relacionada ao desenvolvimento centrado na qualidade de vida, desde melhorias de saúde e educação, até o desenvolvimento de políticas públicas inclusivas.

A sustentabilidade social deve ser avaliada conforme os interesses de todas as populações e seus principais indicadores, dentro de organizações, englobam os recursos humanos internos, a população externa, a participação das partes interessadas e os aspectos macrossociais (LOURENÇO; CARVALHO, 2013).

Embora os pilares da sustentabilidade sejam ambien- 
tal, econômico e social, é importante esclarecer que eles não devem ser analisados individualmente por apresentarem o mesmo grau de importância. Vale ressaltar que o presente estudo foi pautado no pilar da sustentabilidade social. Decidiu-se por este enfoque no intuito de contribuir para os estudos a respeito da dimensão social e buscou-se entender como ela pode ser identificada no Guia Global da Cidade Amiga do Idoso e como as disciplinas do design podem trazer contribuições.

\section{4.}

DESIGN

UNIVERSAL
Acredita-se que o design universal (DU) seja uma das áreas de estudo do design que possa contribuir para a presente discussão. Ele surgiu a partir de debates sobre a redução de barreiras físicas e sobre as necessidades de acesso que começaram a surgir na década de 6o. O DU evoluiu da concepção de um design livre de barreiras, voltado para pessoas com deficiências e com mobilidade reduzida, para um design que atende a todas as pessoas, não importando as suas habilidades e condições físicas (CARLETTO; CAMBIAGHI, 2008; PORTO; REZENDE, 2016).

A Associação Brasileira de Normas Técnicas, na NBR 9050:2015, define o desenho universal como "concepção de produtos, ambientes, programas e serviços a serem utilizados por todas as pessoas, sem necessidade de adaptação ou projeto específco [sic], incluindo os recursos de tecnologia assistiva" (ABNT, 2015, p. 4). Esta definição corrobora a de outros autores acerca do design universal, como a de Mace, Hardie e Place (1991), que o definem como o projeto de todos os produtos, prédios e ambientes externos de forma a poderem ser utilizados pela maior parte das pessoas. 
Ronald Mace foi um dos arquitetos responsáveis por popularizar o termo DU e era diretor do Centro de Design Universal da Universidade do Estado da Carolina do Norte (BOWE, 2000). Este centro é referência no estudo do DU e seus pesquisadores, dentre eles arquitetos, engenheiros e designers, estabeleceram sete princípios que guiam os projetos universais. São eles: uso equitativo, que pode ser utilizado por pessoas com habilidades variadas; uso flexível, que se adapta às necessidades dos diferentes usuários; uso simples e intuitivo, que é fácil de aprender como manusear; informação de fácil percepção, que é fácil de identificar e compreender; tolerância ao erro, que prevê o uso incorreto e previne acidentes; baixo esforço físico, que evita o cansaço após o uso; e dimensões e espaço para aproximação e uso, que permite o uso independentemente das dimensões e alcances dos usuários (CARLETTO; CAMBIAGHI, 2008; NORTH CAROLINA STATE UNIVERSITY, 1997; PORTO, REZENDE, 2016).
5.

RESULTADOSE

DISCUSSÕES
O Guia Global da Cidade Amiga do Idoso traz um pouco dos resultados adquiridos com as entrevistas em grupos focais realizadas nas primeiras 33 cidades que aplicaram o protocolo de Vancouver. Dentre as informações fornecidas constam alguns trechos das entrevistas e interpretações dos resultados (OMS, 2008).

Foi interessante observar que muitas das reclamações e solicitações dos idosos podem ser diretamente relacionadas a soluções sustentáveis. Desta forma, este estudo abordará três dos oito tópicos trazidos pelo Guia Global das Cidades Amigas do Idoso e os relacionará com estudos sobre sustentabilidade social, para refletir sobre a necessidade que os idosos têm por soluções sustentáveis, mesmo que não sejam necessidades declaradas. Os tópicos aqui abordados foram os três primeiros do guia: (1) espaços abertos $\boldsymbol{e}$ prédios; (2) transporte e (3) moradia. Esta escolha foi feita porque estes apresentam interfaces mais concretas de interação com os idosos, comparado aos demais tópicos. 


\section{ESPAÇOS ABERTOS E PRÉDIOS}

As principais questões pontuadas como importantes pelos idosos foram a acessibilidade, a segurança e a qualidade de vida, pois os espaços abertos e prédios interferem em sua mobilidade e independência. Em todas as cidades, a acessibilidade foi um problema recorrente. As barreiras físicas frequentemente desanimam os idosos a saírem de suas casas, restringindo o seu ir e vir (OMS, 2008).

A acessibilidade é uma maneira de garantir a participação das pessoas nas atividades diárias em um ambiente construído de maneira segura, autônoma e confortável. Quando alcançada de forma plena, ela se torna um fator preponderante para a qualidade de vida dos cidadãos, porque garante maior inclusão. A melhoria de acesso é um tópico estratégico na evolução para uma sociedade onde todos participem com suas peculiaridades individuais (FRANCISCO; MENEZES, 2011; FURLANETTO et al., 2013). Isto corrobora a ideia de sustentabilidade social trazida por Barbosa (2008), Faria (2014) e Lourenço e Carvalho (2013). Também se relaciona com a proposta do design universal de Mace, Hardie e Place (1991), Carletto e Cambiaghi (2008) e Porto e Rezende (2016).

Uma prerrogativa do DU é poder favorecer o design inclusivo, que é uma estratégia socioeconomicamente sustentável (SCATOLIM; SANTOS; LANDIM, 2014). Por isso é importante compreender os espaços públicos da cidade e adequá-los aos princípios do DU, aproximando-se de uma maior sustentabilidade na cidade, principalmente em sua dimensão social (SILVA; RODRIGUES, 2007).

No que se refere aos prédios, é necessário que sejam mais amigáveis aos idosos apresentando, por exemplo, elevadores, rampas, portas e corredores amplos, escadas adequadas e com corrimão, piso antiderrapante, sinalização eficiente, áreas para descanso e banheiros públicos acessíveis (OMS, 2008). Estas soluções são, também, fortemente recomendadas pelo design universal através dos seus sete princípios. Eles fornecem embasamento para a criação de soluções satisfatórias para uma ampla gama de pessoas (CARLETTO; CAMBIAGHI, 2008; GAIA, 2005).

\section{TRANSPORTE}

Este tópico engloba os aspectos de infraestrutura, de equipamentos e de serviços para os meios de transporte urbanos, entendendo que a existência de transporte público ou privado eficiente e com baixo custo é essencial para o envelhecimento ativo (OMS, 2008). Conforme Campos (2006), sob a perspectiva da sustentabilidade, a mobilidade pode ser tratada tanto pela adequação dos meios de transporte aos usuários e seus contextos socioeconômicos, quanto pelas questões de qualidade ambiental.

São diversos os meios de transporte existentes nas cidades, desde ônibus e metrôs até carros com motoristas privados. Algumas das reclamações dos idosos se referem ao reduzido número de ônibus, o que prejudica o conforto e a segurança dos idosos, porque 
favorece a superlotação. Ocorre, também, de o itinerário dos transportes públicos não atenderem bem às necessidades dos idosos, pois não os levam aos destinos que lhes são importantes, tais como, instituições de longa permanência e jardins públicos (OMS, 2008).

É interessante que a oferta de transporte público seja maximizada e que a cidade seja descentralizada em subcentros. Desta forma, os trajetos realizados poderão ser reduzidos em distância e tempo e os subcentros poderão ser mais bem servidos de coletivos, aprimorando a qualidade de vida dos cidadãos (ACSELRAD, 1999).

Sobre os veículos, o embarque e o desembarque são tidos como grave problema. Diversas cidades já atendem a esta questão com pisos rebaixados nos ônibus e adaptações que facilitam o embarque e o desembarque. Entretanto, outras apresentam veículos antigos, danificados e sem acesso para cadeirantes (OMS, 2008).

Já foi mencionada a importância da acessibilidade para a sustentabilidade social e, no que se refere ao transporte, ela também se mantém. Conforme Silva e Rodrigues (2007), quando a acessibilidade é fraca, a sustentabilidade pode ser comprometida. Ademais, Campos (2006) afirma que deve existir uma preocupação quanto ao veículo utilizado. Além da necessidade de reduzir a poluição, com o uso de veículos tecnologicamente mais sustentáveis, as vias e locais de paradas devem atender bem aos usuários, o que ajuda a manter a fluidez do trânsito. $\mathrm{O}$ transporte privado, por sua vez, gera maiores danos ambientais, quando o foco é a qualidade do ar. Por isso, é interessante que ele seja reduzido na medida em que o transporte público ou as vias para pedestres e ciclistas são melhorados.

A atitude do jovem de ceder lugar nos transportes públicos ainda não é geral, assim como a gentileza do motorista e a sensibilidade com relação aos idosos. Sugere-se esperar que o idoso sente antes de arrancar o ônibus, evitando desequilíbrios e quedas (OMS, 2008). Estas gentilezas urbanas são responsáveis por aprimorar o bem-estar do idoso e reduzir o uso de transporte privado. Mais uma vez a sustentabilidade social pautada na qualidade de vida de Barbosa (2008) e Faria (2014) pode ser identificada.

Quanto aos estacionamentos, os idosos consideram-nos uma característica amigável, quando são disponíveis para eles e de fácil acesso para deficientes (OMS, 2008). Micheletto (2011) explica que, no Brasil, o Estatuto do Idoso prevê que 5\% das vagas dos estacionamentos públicos e privados sejam destinadas a idosos, estando, ainda, posicionadas em local mais cômodo ao usuário. Além disso, a respeito dos estacionamentos rotativos, Quadros et al. (2013) esclarecem que eles refletem a sustentabilidade por permitir que mais pessoas utilizem as vagas. Ao reduzir o tempo de permanência, este método gera melhorias ao trânsito, estimula o comércio, aumenta o dinamismo no local, gera maior fluidez e, consequentemente, traz melhorias para o uso das vagas e para a qualidade de vida. 


\section{MORADIA}

A questão das moradias é essencial para o bem-estar e para a segurança do idoso. Muitos dos problemas elencados se referem a custos e gastos com as casas, desde o aluguel até a manutenção e adaptação. $\mathrm{O}$ acesso fácil e rápido a serviços, a proximidade com os familiares e a continuidade da participação na comunidade são largamente valorizados. A perda destes vínculos é tida como uma característica não amigável (OMS, 2008).

Ante estas questões, ressalta-se aqui o conceito aging-in-place, que significa envelhecer no próprio lar e na comunidade com segurança, independência e conforto, não importando a idade, condições financeiras ou capacidades físicas. Como as moradias não se limitam à casa e à comunidade, o aging-in-place considera também questões como: sustentabilidade, serviços e mobilidade. Ao instigar a permanência do idoso em sua casa, este conceito ajuda a evitar gastos com instituições públicas. Portanto, também representa um importante e interessante ponto de reflexão para entidades políticas e de saúde (PORTO; REZENDE, 2016). É importante destacar o estudo de Arigoni, Ceccon e Damazio (2016), que evidencia a tendência que existe de os idosos morarem sozinhos e desejarem manter sua autonomia. As autoras ressaltam a relevância do design para gerar soluções que favoreçam um envelhecimento com maior qualidade.

Outras situações apontadas pelos idosos sobre as moradias dizem respeito ao planejamento visando garantir a acessibilidade. É importante que seus lares sejam construídos com materiais adequados, evitando superfícies desniveladas; com disponibilidade para instalação de elevador, se necessário; com banheiros e cozinhas adaptados; espaços amplos para uma boa locomoção, dentre outras recomendações (OMS, 2008). Como mencionado anteriormente, muitos destes problemas são atendidos pelo DU. Ele também garante que os idosos possam envelhecer em seus lares, pois gera soluções facilmente utilizadas por pessoas com idades, habilidades e características variadas. Desta maneira, mudar e adaptar os lares para pessoas idosas ou não idosas e construí-los com enfoque no design para os usuários pode agregar muitos benefícios para os moradores (CREWS; ZAVOTKA, 2006; KOCHERA; STRAIGHT; GUTERBOCK, 2005; PORTO; REZENDE, 2016).

Os três tópicos explicados até aqui apresentam questões muitas vezes de interesse público. Mesmo as moradias, não se restringem ao interior das residências, o que faz com que os meios externos e a comunidade também interfiram no bem-estar dos idosos. Águas (2012) explica que os espaços públicos privilegiam a socialização e a expressão social. Por esta razão devem ser frequentados a fim de incentivar a vida social entre as pessoas, fomentando o exercício da cidadania.

A metodologia utilizada pelo Guia Global da Cidade Amiga do Idoso, como mencionado anteriormente, utiliza uma abordagem bottom-up, ou seja, ela valoriza as opiniões dos verdadeiramente interessados 
nas mudanças, no caso, os idosos (OMS, 2008). No design, as abordagens participativas estão preocupadas em entender a fundo as necessidades de todas as partes interessadas no projeto, a fim de gerar soluções que realmente agradem a todos, corroborando o que Lourenço e Carvalho (2013) defendem a respeito da sustentabilidade social. Neste sentido, métodos participativos de design se voltam para incluir os interessados em seu desenvolvimento como vozes ativas, ou como parceiros do projeto (ÁGUAS, 2012).

Para projetos de design universal, também é sugerido o envolvimento dos usuários, com características diversas, em todas as etapas do projeto, com o objetivo de atender às expectativas da maior quantidade de indivíduos possível (BURGSTAHLER, 2009; RAVISELVAM; NOONAN; HÖLTTÄ-OTTO, 2014).

Acredita-se, portanto, que trabalhar as necessidades e os desejos dos idosos nas cidades com a sua participação direta seja uma maneira de promover a sustentabilidade social. O uso do DU com abordagens mais participativas pode criar soluções não apenas para os idosos, mas para que toda a população envelheça com acessibilidade e qualidade de vida.

\section{6.}

CONSIDERA-

ÇÕES FINAIS
Com o crescimento da população idosa muitos problemas antes despercebidos passaram a ser foco de debates para melhorias na qualidade de vida do idoso. O Guia Global da Cidade Amiga do Idoso incentiva avanços em prol de um envelhecimento ativo e avalia as condições que as cidades proporcionam para os seus habitantes com idade superior aos sessenta anos. Após a entrevista com os idosos, em grupos focais, têm-se informações sobre suas necessidades, desejos e questões que precisam ser melhoradas nas cidades. É importante que estas opiniões, vindas direto dos principais interessados, sejam analisadas e levadas aos poderes públicos.

Pode-se observar uma relação entre os três primeiros itens do Guia Global da Cidade Amiga do Idoso (espaços abertos e prédios, transporte e moradia) e as premissas da sustentabilidade social. Além disso, também se identificou que o DU representa uma ótima contribuição para buscar soluções que sejam pertinentes às questões do guia. Desta forma, a inserção do design neste contexto se dá pela sua característica de visualizar os problemas de forma holística, 
considerando a participação dos usuários e utilizando a criatividade para alcançar melhores soluções. Estes elementos podem ser fortes aliados na oferta de subsídios para a formulação de políticas públicas e ações em benefício da população idosa e da sustentabilidade social.

Em vista disso, a aplicação do Guia Global da Cidade Amiga do Idoso poderá ser enriquecida caso sejam consideradas as questões de sustentabilidade, as desejadas pelos idosos e as contribuições que o design tem a oferecer. Esta forma de aplicação também fornece perspectivas de estudos acadêmicos mais pautados no contexto de cada local e na participação ativa dos idosos. Por esta razão, são sugeridos mais estudos e novas experiências acerca do guia com o apoio do design e fundamentado pela sustentabilidade.

\section{REFERÊNCIAS ACSELRAD, H. Discursos da sustentabilidade} urbana. Revista Brasileira de Estudos Urbanos e Regionais, Recife, n. 1, p. 79-90, 1999.

ÁGUAS, S. Do design ao co-design. Uma oportunidade de design participativo na transformação do espaço público. On the waterfront, Barcelona, v. 22, p. 57- 70, 2012.

ALVES, M. J. Mobilidade e acessibilidade: conceitos e novas práticas. Indústria e ambientes, Porto, n. 55, p. 12-14, 2006.

ARIGONI, L. B.; CECCON, M.; DAMAZIO, V. Morar com mais independência e autonomia: uma reflexão sobre a perspectiva do design. In: CONGRESSO

BRASILEIRO DE PESQUISA E DESENVOLVIMENTO EM DESIGN. 12. 2016. Belo Horizonte. Anais... São Paulo: Blücher, 2016, 10p.

ASSOCIAÇÃO BRASILEIRA DE NORMAS TÉCNICAS. NBR 9050: Acessibilidade a edificações, mobiliário, espaços e equipamentos urbanos. Rio de Janeiro, 2015a. 
BARBOSA, G. S. O desafio do desenvolvimento sustentável. Revista Visões, v. 1, n. 4, p.1-11, 2008.

BESTETTI, M. L. T.; GRAEFF, B.; DOMINGUES, M. A. O impacto da urbanidade no envelhecimento humano: o que podemos aprender com a estratégia Cidade Amiga do Idoso? Revista temática Kairós Gerontologia, v. 15, n. 6, p. $117-136$, 2012.

BOWE, F. G. Universal design in education: teaching non-traditional students. Westport: Bergin \& Garvey, 2000, $133 \mathrm{p}$.

BURGSTAHLER, S. Universal Design: process, principles, and applications. University of Washington. Seattle. p. 1-4, 2009. Disponível em: < https://bit.ly/1wZOOkW>. Acesso em 10 de maio de 2017.

CAMPOS, V. B. G. Uma Visão da Mobilidade Urbana Sustentável. Revista dos Transportes Públicos, São Paulo, 2006.

CARletto, A. C.; CAMBIAghi, S. Desenho Universal: um conceito para todos. São Paulo: Instituto Mara Gabrilli, 2008.

CHICK, A. Design for social innovation: emerging principles and approaches. Iridescent: Icograda Journal of Design Research, v. 2, n. 1, 2012.

CREWS, D. E.; ZAVOTKA, S. Aging disability, and frailty: implications for universal design. Journal of Physiological Anthropology, v. 25, p. 113 118, 2066.

ELKINGTON, J. Enter the Triple Bottom Line. In: HENRIQUES, A.; RICHARDSON, J. The triple bottom line. Does it all add up? Londres: Earthscan, 2004. Cap. 1, p. 1-16.

FARIA, J. H de. Por uma teoria crítica da sustentabilidade. Organizações e Sustentabilidade, Londrina, v. 2, n. 1, p. 2 - 25, 2014.

FRANCISCO, P. C. M.; MENEZES, A. M. Design universal, acessibilidade e espaço construído. Construindo, Belo Horizonte, v. 3, n. 1, p. 25-29, 2011.

FREITAS, R. F. de; COUTINHO, S. G.; WAECHTER, H. N. Análise de metodologias em design: a informação tratada por diferentes olhares. Estudos em design. Rio de Janeiro, v. 21, n. 1, p. 1-15, 2013.

FURLANETTO, C. et al. Qualidade urbana e mobilidade: condições de acessibilidade em habitação de interesse social. Revista brasileira de ergonomia, Rio de Janeiro,v. 8, n. 2, p. 1-18, 2013.

GAIA, S. Habitações de interesse social para a terceira idade sob a ótica dos princípios de acessibilidade promovidos pelo Desenho Universal: o idoso e suas necessidades específicas. 2005. 183 f. Dissertação (Mestrado em Cons- 
trução Civil) - Universidade Federal do Paraná, Curitiba, 2005.

GRAEFF, B.; DOMINGUES, M. A.; BESTETTI, M. L. T. Bairro amigo do idoso no Brás: percepções sobre os migrantes internacionais. Revista temática Kairós Gerontologia. São Paulo, v. 15, n. 6, p. 177-196, 2012.

JORDÃO, H. M.; SILVA, M. R. C. e. Intervenções urbanas e suas precariedades. Estudos Vida e Saúde, Goiânia, v. 41, n. especial, p. 81 - 92, 2014.

KOCHERA, A.; STRAIGHT, A.; GUTERBOCK, T. Beyond 50.05: a report to the nation on livable communities. Denver: AARP Public Policy Institute, 2005. p. 52-75. Report.

LOURENÇO, M. L.; CARVALHO, D. Sustentabilidade social e desenvolvimento sustentável. Revista de Administração, Contabilidade e Economia, Joaçaba, v. 12, n. 1, p. 9 - 38, 2013.

MACE R. L.; HARDIE, G. J.; PLACE, J. P. Accessible environments: toward universal design. Raleigh: The Center for Universal Design, North Carolina State University, 1991. 44 p.

MICHELETTO, T. M. G. P. O risco do idoso pedestre nas vias urbanas. Companhia de engenharia de tráfego, São Paulo, notas técnicas, p.1-13, 2011.

MONTEIRO, L. C.; ZAZZETTA，M. S.; ARAÚJO
JÚNIOR, M. E. Sustentabilidade: Relações entre espaço urbano e envelhecimento ativo. Revista Novos Estudos Jurídicos, Itajaí, v. 20, n. 1, p. 116- 145, 2015.

MORAIS, D. et al. Teoria da Atividade para Entendimento de Práticas Humanas no Desenvolvimento Participativo de Jogos. In: CONGRESSO INTERNACIONAL INFORMÁTICA EDUCATIVA. 19. 2014. Anais... Fortaleza: TISE, p. 815-820, 2014.

NASRI, F. O envelhecimento populacional no Brasil. Einstein, São Paulo, v. 6, n. supl. 1, p. s4-s6, 2008.

NORTH CAROLINA STATE UNIVERSITY CENTER FOR UNIVERSAL DESIGN. The principles of universal design. 1997. Disponível em: <https:// bit.ly/2rLnBIA> . Acesso em 10 de maio de 2014.

OLIVEIRA, J. H. S. et al. Envelhecer com qualidade? Reflexo de ações extensionistas em instituições asilares. Revista Ciência em Extensão, São Paulo, v. 12, n.2, p. 141-152, 2016.

ORGANIZAÇÃO MUNDIAL DE SAÚDE. Envelhecimento ativo: uma política de saúde. Brasília: Organização Pan-Americana da Saúde. 2005. 6op.

ORGANIZAÇÃO MUNDIAL DE SAÚDE. Guia Global: cidade amiga do idoso. Genebra: Organização Mundial da Saúde. 2008. 67p. 
PEDRO, M. S. B. et al. Políticas públicas para o envelhecimento ativo. In: ENCONTRO INTERNACIONAL PARTICIPAÇÃO, DEMOCRACIA E POLÍTICAS PÚBLICAS: APROXIMANDO AGENDAS E AGENTES. 2013. Anais... Araraquara: UNESP, 24 pp., 2013.

PORTO, C. F.; REZENDE, E. J. C. Terceira idade, design universal e aging-in-place. Estudos em Design, Rio de Janeiro, v. 24, n. 1, p. 152-168, 2016.

QUADROS, C. et al. Mobilidade Urbana em Rio Grande - RS: uma visão interdisciplinar. In: SIMPÓSIO INTERNACIONAL SOBRE INTERDISCIPLINARIDADE NO ENSINO, NA PESQUISA E NA EXTENSÃO - REGIÃO SUL. 2013. Florianópolis. Anais... Florianópolis: EGC, 2013, p. 759-768.

RAVISELVAM, S.; NOONAN, M; HÖLTTA-OTTO, $K$. Using elderly as lead users for universal engineering design. In: UNIVERSAL DESIGN 2014: THREE DAYS OF CREATIVITY AND DIVERSITY. Proceedings of the international conference on universal design... Lund: IOSpress, 2014. p. 366-375.

RAUTH, J.; SANTOS, E. R.; PEDDE, V. Projeto RS Amigo do Idoso: uma amizade que não se efetivou. Textos e contextos. Porto Alegre, v. 11, n. 1, p. 156-171. 2012.

SCATOLIM, R. L.; SANTOS, J. E. G.; LANDIM, P. C. Aspectos do design universal: uma proposta de máquina de cartão para deficientes visuais. In: CONGRESSO BRASILEIRA DE PESQUISA
E DESENVOLVIMENTO EM DESIGN, 11. 2014. Gramado. Proceedings... São Paulo: Blücher, 2014, 9p.

SILVA, J. C. R. C. E RODRIGUES, J. C. C.Acessibilidade no espaço público urbano: um novo desafio para a sustentabilidade. In: ENCONTRO NACIONAL, 4, E ENCONTRO LATINO AMERICANO SOBRE EDIFICAÇÃO E COMUNIDADES SUSTENTÁVEIS, 2., 2007. Campo Grande. Anais... Campo Grande: ANTAC, 2007. 